\title{
DHEA - Brass Ring or Red Herring?
}

$\mathrm{G}^{\mathrm{a}}$ erontologists want so much to believe in DHEA. Early enthusiasm for DHEA - the celebrity steroid whose exploits are the focus of an impressive proportion of popscience books on cures for aging and the proud possessor of hectares of shelf space in health food stores - seemed to be on fairly steady ground, at least in comparison with the scientific evidence for the antigeric properties attributed to melatonin and royal jelly. Epidemiological evidence suggested associations between low DHEA levels and high relative risks for breast cancer ${ }^{1}$ and cardiovascular disease. ${ }^{2}$ Studies of the metabolic effects of DHEA suggested that it might help to protect against the atherogenic effects of adult-onset diabetes and obesity. ${ }^{3} \mathrm{~A}$ single injection of DHEA into old mice was said to correct many aspects of immunosenescence, including poor primary and secondary antibody responses to neoantigens. ${ }^{4}$ Aging mice given high doses of DHEA in their food were lighter, had lower rates of kidney failure, and were cancer-resistant. ${ }^{5}$ Besides, there was so much of it in young adults, and its levels declined so convincingly in older people, that it just had to do something good, didn't it?

New data and new arguments have begun to temper the excess of enthusiasm for this mysterious steroid. Follow-up studies have shown that high DHEA levels in mid-life do not, in the longer term, predict resistance to mortality from cardiovascular illness in men $^{6}$ or women. ${ }^{7}$ Although high DHEA levels may be associated with resistance to breast cancer among premenopausal women, they seem to confer greater breast cancer risk in postmenopausal women. ${ }^{8,9}$ High levels of DHEA do not seem to convey protection against cognitive failure $^{10}$ or obesity or glucose intolerance. ${ }^{11}$ Thus the epidemiological evidence associating high levels of serum DHEA with resistance to the diseases and disabilities of old age is now tenuous at best.

Replications of the initial dramatic reports of immunorejuvenation in mice have been slow in coming, and interpretation of the rodent data has been complicated by the realization that rodents neither make DHEA nor retain ingested DHEA. Our own ongoing intervention trial of lifelong oral DHEA-S in genetically heterogeneous mice has, to date, shown no evidence for positive effects of this steroid on immunological responses or on survival (Miller et al., unpublished data). Similarly, clinical studies of DHEA as a potentiator of immune responses of middle-aged or older people to vaccination have been disappointing, providing little or no evidence for clinical efficacy in trials using influenza or tetanus vaccines. ${ }^{12,13}$ DHEA administration to postmenopausal women for 3 weeks was found to have no positive effects on $\mathrm{T}$ cell proliferation or cytokine production, although the function of natural killer cells in the blood was increased significantly ${ }^{14}$ in this double-blind cross-over study. A singleblind study in which DHEA was administered daily for 20 weeks to a group of older men (average age 64 years) pro- duced no consistent or convincing changes in $\mathrm{T}$ cell responsiveness; the transient positive results claimed by these authors seem more likely to represent chance fluctuations over a long series of multiple comparisons, ${ }^{15}$ and the study did not include parallel studies of placebo control subjects.

Long-term (6 to 12 month) administration of DHEA to volunteers has little or no effect on objective measures of physiological health. ${ }^{15,16}$ Thus, a 6-month double-blind study of DHEA given at $50 \mathrm{mg} /$ day to 30 volunteers aged 40 to 70 years found no effects on lipid profiles, insulin sensitivity, or body fat; the exception was a small decline in high density lipoprotein levels in women. A second study using DHEA at $100 \mathrm{mg} /$ day for 12 months again found no change in lipid or apolipoprotein levels, lumbar muscle strength, glucose or insulin levels, or bone mineral density. Knee muscle strength increased in the men by about $15 \%$, with the placebo effect responsible for half the change; knee strength decreased in the women. Androgen levels increased significantly among women in both of these studies, exceeding the normal range in the longer study. The only consistent dramatic effect was a psychological one: $67 \%$ of the men and $84 \%$ of the women in the 1 -year study reported improvements in their own sense of well-being while ingesting DHEA.

\section{See also p 1395}

The current status of DHEA research suggests that learning more about the basic biology of steroid action should take precedence over the ever-alluring temptation to rush into clinical trials. Hornsby's review suggests a number of questions whose answers might help to point the way to a more judicious selection of physiological targets for intervention. The distribution among tissues of DHEA sulfatases, the ability of target tissues to transform DHEA into active metabolites, the source of and role for DHEA in the central nervous system, the interactions between DHEA and other steroids whose levels (and actions) may vary with age and gender, and the potential role of DHEA in peroxisome regulation - each of these areas is likely to reward additional investigation, and could help to guide a second, and possibly more productive, wave of clinical investigations.

Hornsby's review also provides an admirable example of the place for comparative biology in experimental aging research. One implication is fairly obvious: those of us who work in animal models need to remain watchful for exceptions to our tacit assumption that mice are simply small nocturnal people with prominent whiskers. Much of what we learn about the biochemistry, physiology, neurobiology, pathology, and genetics of mice, rats, dogs, and cats can be applied usefully, with appropriate caution and confirmation, to human biology, but much of it cannot. It is not simply that reports of DHEA administration to rats and mice will need to 
be re-labeled "pharmacology" rather than "hormonereplacement" studies, but rather that investigations of the action of this steroid in animals will need to take note of species-specific peculiarities in its uptake, transport, metabolism, excretion, and cellular actions, which may be very different in species that differ more than 100 -fold in the natural levels of circulating DHEA. (The demonstration that nearly all common strains of laboratory mice lack the enzymes needed to synthesize melatonin ${ }^{17}$ has led to a similar reassessment of the reported effects of pineal transplants and melatonin inhibitors in aging mice.)

The second implication is less discouraging: the restriction of DHEA to humans, chimps, and their close relatives, while arguing against an important general role of DHEA in mammalian aging, is consistent with the idea that this hormone may indeed play a key role in the timing of human life-history events. Evolution of long-lived primates has involved strong selection for genes that delay sexual maturation and for genes that postpone diseases to ages beyond those of full reproductive maturity. Physiological traits that sharply distinguish humans from their shorter-lived cousins such as the unexplained predominance of DHEA among circulating human steroids, and its careful age-dependent regulation - may provide key clues as to how these antigeriatric tricks are accomplished.

Richard A. Miller University of Michigan

\section{REFERENCES}

1. Bulbrook RD, Hayward JL, Spicer CC. Relation between urinary androgen and corticoid secretion and subsequent breast cancer. Lancet 1971;2:395398.

2. Barrett-Conner E, Khaw K-T, Yen SSC. A prospective study of dehydroepiandrosterone sulfate, mortality, and cardiovascular disease. N Engl J Med 1986;315:1519-1524.

3. Nestler JE, Clore JN, Blackard WG. Dehydroepiandrosterone: The "missing link" between hyperinsulinemia and atherosclerosis? FASEB J 1992;6:30733075 .
4. Daynes RA, Araneo BA. Prevention and reversal of some age-associated changes in immunologic responses by supplemental dehydroepiandrosterone sulfate therapy. Aging Immunol Infect Dis 1992;3:135-154.

5. Schwartz AG, Whitcomb JM, Nyce JW et al. Dehydroepiandrosterone and structural analogs: A new class of cancer chemopreventive agents. Adv Cancer Res 1988;51:391-424.

6. Barrett-Connor E, Goodman-Gruen D. The epidemiology of DHEAS and cardiovascular disease. Ann N Y Acad Sci 1995;774:259-70:259-270.

7. Barrett-Connor E, Goodman-Gruen D. Dehydroepiandrosterone sulfate does not predict cardiovascular death in postmenopausal women. The Rancho Bernardo Study. Circulation 1995;91:1757-1760.

8. Zumoff B, Levin J, Rosenfeld RS et al. Abnormal 24-hr mean plasma concentrations of dehydroisoandrosterone and dehydroisoandrosterone sulfate in women with primaty operable breast cancer. Cancer Res 1981;41:33603363.

9. Gordon GB, Bush TL, Helzlsouer KJ et al. Relationship of serum levels of dehydroepiandrosterone and dehydroepiandrosterone sulfate to the risk of developing postmenopausal breast cancer. Cancer Res 1990;50:3859-3862.

10. Barrett-Connor E, Edelstein SL. A prospective study of dehydroepiandrosterone sulfate and cognitive function in an older population: The Rancho Bernardo Study. J Am Geriatr Soc 1994;42:420-423.

11. Barrett-Connor E, Ferrara A. Dehydroepiandrosterone, dehydroepiandrosterone sulfate, obesity, waist-hip ratio, and noninsulin-dependent diabetes in postmenopausal women: the Rancho Bernardo Study. J Clin Endocrinol Metab 1996;81:59-64.

12. Araneo B, Dowell T, Woods ML et al. DHEAS as an effective vaccine adjuvant in elderly humans. Proof-of-principle studies. Ann N Y Acad Sci 1995;774:232-48:232-248.

13. Degelau J, Guay D, Hallgren $H$. The effect of DHEAS on influenza vaccination in aging adults. J Am Geriatr Soc 1997;45:747-751.

14. Casson PR, Andersen RN, Herrod HG et al. Oral dehydroepiandrosterone in physiologic doses modulates immune function in postmenopausal women. Am J Obstet Gynecol 1993;169:1.536-1539.

15. Yen SS, Morales AJ, Khorram O. Replacement of DHEA in aging men and women. Potential remedial effects. Ann N Y Acad Sci 1995;774:128-42:128-142.

16. Morales AJ, Nolan JJ, Nelson JC et al. Effects of replacement dose of dehydroepiandrosterone in men and women of advancing age [published erratum appears in J Clin Endocrinol Metab 1995 Sep;80:2799]. J Clin Endocrinol Metab 1994;78:1360-1367.

17. Ebihara S, Marks T, Hudson DJ et al. Genetic control of melatonin synthesis in the pineal gland of the mouse. Science 1986;231:491-493. 